\title{
Recent Progress in the Understanding of the $r$-Process
}

\author{
Yong-Zhong Qian ${ }^{* \dagger}$ \\ School of Physics and Astronomy, University of Minnesota, Minneapolis, MN 55455, USA \\ E-mail: qian@physics.umn.edu
}

\begin{abstract}
A brief overview of the $r$-process is given with an emphasis on the observational implications for this process. The conditions required for the major production of the heavy $r$-process elements (r-elements) with mass numbers $A>130$ are discussed based on a generic astrophysical model where matter adiabatically expands from a hot and dense initial state. Nucleosynthesis in the neutrino-driven winds from nascent neutron stars is discussed as a specific example. Such winds readily produce the elements from $\mathrm{Sr}$ to $\mathrm{Ag}$ with $A \sim 88-110$ through charged-particle reactions in the $\alpha$-process but appear incapable of making the heavy $r$-elements. Observations of elemental abundances in metal-poor stars have provided many valuable insights into the $r$-process. They have demonstrated that the production of the heavy $r$-elements must be associated with massive stars evolving on short timescales, provided evidence strongly favoring core-collapse supernovae over neutron star mergers as the major source for these elements, and shown that this source cannot produce any significant amount of the low-A elements from $\mathrm{Na}$ to $\mathrm{Ge}$ including $\mathrm{Fe}$. A self-consistent astrophysical model of the $r$-process remains to be developed, and it appears well worthwhile to carry out more comprehensive studies on the evolution and explosion of the massive stars of $\sim 8-11 M_{\odot}$ that undergo $\mathrm{O}-\mathrm{Ne}-\mathrm{Mg}$ core collapse and produce a very insignificant amount of the low- $A$ elements.
\end{abstract}

10th Symposium on Nuclei in the Cosmos

July 27 - August 1, 2008

Mackinac Island, Michigan, USA

\footnotetext{
${ }^{*}$ Speaker.

${ }^{\dagger}$ This work was supported in part by US DOE grant DE-FG02-87ER40328.
} 


\section{Introduction}

This paper discusses some of the progress that has been made in the understanding of the $r$-process since the publication of [1], a major review on this process, in 1991. More detailed discussion can be found in [2,3]. Readers are referred to [4] for a review of the $r$-process that covers a wider range of topics and has different emphases.

For convenience of discussion, an $r$-process event can be separated into two phases. During the first phase, relatively heavy nuclei are produced mostly via charged-particle reactions (CPRs). These nuclei become the seed nuclei to capture the neutrons during the second phase. The crucial result of the first phase is a large (number) abundance ratio of the neutrons to the seed nuclei. The second phase involves neutron-rich nuclei far from stability participating in a number of types of nuclear reactions, which include neutron capture, photo-disintegration, $\beta$-decay, and possibly fission. The properties of such nuclei play an essential role in determining the exact abundance pattern produced by the $r$-process event. This important aspect of the $r$-process will not be discussed here. For the presentation below, the abundance pattern resulting from the second phase is characterized by the average mass number $\left\langle A_{r}\right\rangle$ of the corresponding $r$-process nuclei. Assuming that all the neutrons are captured, the results from the first and second phases are related by mass conservation as

$$
\left\langle A_{s}\right\rangle Y_{s}+Y_{n}=\left\langle A_{r}\right\rangle Y_{r},
$$

where $\left\langle A_{s}\right\rangle$ is the average mass number of the seed nuclei, $Y_{s}$ is their initial total (number) abundance, $Y_{n}$ is the initial neutron abundance, and $Y_{r}$ is the final total abundance of the $r$-process nuclei produced. In the absence of fission, the total number of heavy nuclei is conserved. For this case $Y_{r}=Y_{S}$ and Equation (1.1) can be rewritten as

$$
\left\langle A_{s}\right\rangle+\frac{Y_{n}}{Y_{s}}=\left\langle A_{r}\right\rangle
$$

which relates the average $r$-process nuclei to the average seed nuclei by the neutron-to-seed ratio $Y_{n} / Y_{s}$. Extensive fission cycling occurs for $Y_{n} / Y_{s} \gg\left\langle A_{s}\right\rangle$, in which case Equation (1.1) approximately becomes

$$
Y_{n} \approx\left\langle A_{r}\right\rangle Y_{r}
$$

For this extreme case, a regular abundance pattern is expected from the steady-state nature of the nuclear flow between the fission products and the fissioning nuclei, and the final outcome is governed by the initial neutron abundance but not the exact abundance or form of the seed nuclei.

It can be seen from the above discussion that the neutron-to-seed ratio $Y_{n} / Y_{s}$ is crucial to the success of an $r$-process event. Before discussing how this ratio is determined in the astrophysical models of these events, some general comments regarding the $r$-process based on observations are in order. Figure 1 shows the $\log \varepsilon$ values $^{1}$ for the elements from $\mathrm{Sr}$ to $\mathrm{Ag}$ with mass numbers $A \sim 88-110$ and those from Ba to Au with $A>130$ observed in four metal-poor stars CS 22892 052 [5], HD 221170 [6], CS 31082-001 [7], and BD $+17^{\circ} 3248$ [8] with $[\mathrm{Fe} / \mathrm{H}] \equiv \log (\mathrm{Fe} / \mathrm{H})-$ $(\mathrm{Fe} / \mathrm{H})_{\odot}=-3.1,-2.2,-2.9$, and -2.1 , respectively. The solid curves in Fgiures $1 \mathrm{a}$ and $1 \mathrm{~b}$ represent the so-called solar " $r$-process" abundance pattern ( $r$-pattern) that is translated to pass

\footnotetext{
${ }^{1}$ For an element $\mathrm{E}$ with a (number) abundance ratio $(\mathrm{E} / \mathrm{H})$ relative to hydrogen, $\log \varepsilon(\mathrm{E}) \equiv \log (\mathrm{E} / \mathrm{H})+12$.
} 

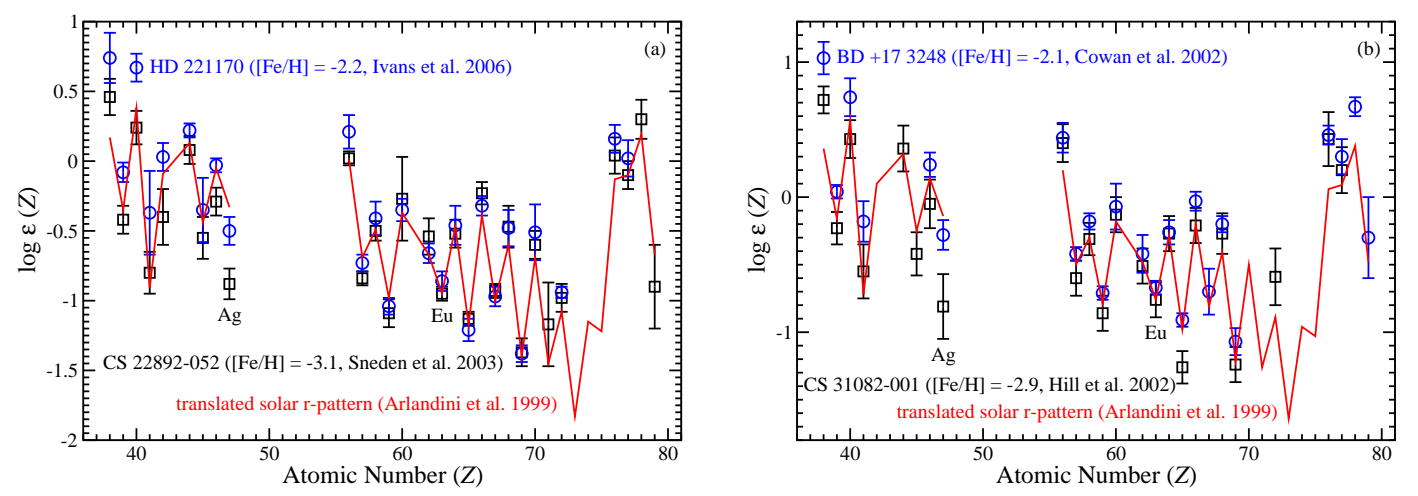

Figure 1: Data on the $\log \varepsilon$ values for the CPR elements from $\mathrm{Sr}$ to $\mathrm{Ag}(Z=38-47, A \sim 88-110)$ and the heavy $r$-elements from $\mathrm{Ba}$ to $\mathrm{Au}(Z=56-79, A>130)$ for (a) CS 22892-052 (squares with error bars; [5]) and HD 221170 (circles with error bars; [6]) and (b) CS 31082-001 (squares with error bars; [7]) and $\mathrm{BD}+17^{\circ} 3248$ (circles with error bars; [8]). The solid curves in (a) and (b) represent the solar " $r$-pattern" [9] that is translated to pass through the Eu data for CS 22892-052 and CS 31082-001, respectively. Note that the data on the heavy $r$-elements follow the translated solar $r$-pattern rather well, especially for the two stars in (a). However, the CPR elements, especially $\mathrm{Sr}$ and Ag, exhibit significant to large deviations. As discussed in Section 2.1, these elements are not the true $r$-elements produced by rapid neutron capture.

through the Eu data for CS 22892-052 and CS 31082-001, respectively. This pattern was derived by subtracting the $s$-process contributions calculated in [9] from the total solar abundances. It can be seen from Figure 1 that the data for the elements with $A>130$ (Ba to $\mathrm{Au}$ with atomic numbers $Z=56-79)$ in all four stars follow the translated solar $r$-pattern rather well, especially for the two stars in Figure 1a. These elements will be referred to as the heavy $r$-process elements ( $r$-elements) hereafter. However, the data for the elements with $A \sim 88-110$, especially $\operatorname{Sr}$ and $\operatorname{Ag}(Z=38$ and 47, respectively), exhibit significant to large deviations. As will be discussed in Section 2.1, these elements are routinely produced by the CPRs in the neutrino-driven wind from a newlyformed neutron star, and therefore, are not the true $r$-elements produced by rapid neutron capture. They will be referred to as the CPR elements hereafter. The remarkable agreement between the solar $r$-pattern and the data for the heavy $r$-elements in four metal-poor stars demonstrates that the $r$-process already occurred in the early Galaxy, and therefore, must be associated with massive stars evolving on short timescales. The rather regular abundance pattern exhibited by the heavy $r$-elements also suggests that fission cycling may be involved in their production.

\section{Modeling and understanding the $r$-process}

A generic model of the $r$-process is associated with expansion of matter as it is ejected from a hot and dense initial state inside some astrophysical environment. At initial temperatures of $\gtrsim 1 \mathrm{MeV}$, this matter is simply composed of free neutrons and protons with their abundances specified by the electron fraction $Y_{e}$ (matter is neutron-rich for $Y_{e}<0.5$ ). The nucleosynthesis in this matter depends on the evolution of its temperature $T$ and density $\rho$ with time $t$ during the subsequent expansion. Assuming an adiabatic expansion with $T(t) \propto \exp \left(-t / \tau_{\mathrm{dyn}}\right)$, where $\tau_{\mathrm{dyn}}$ is a 


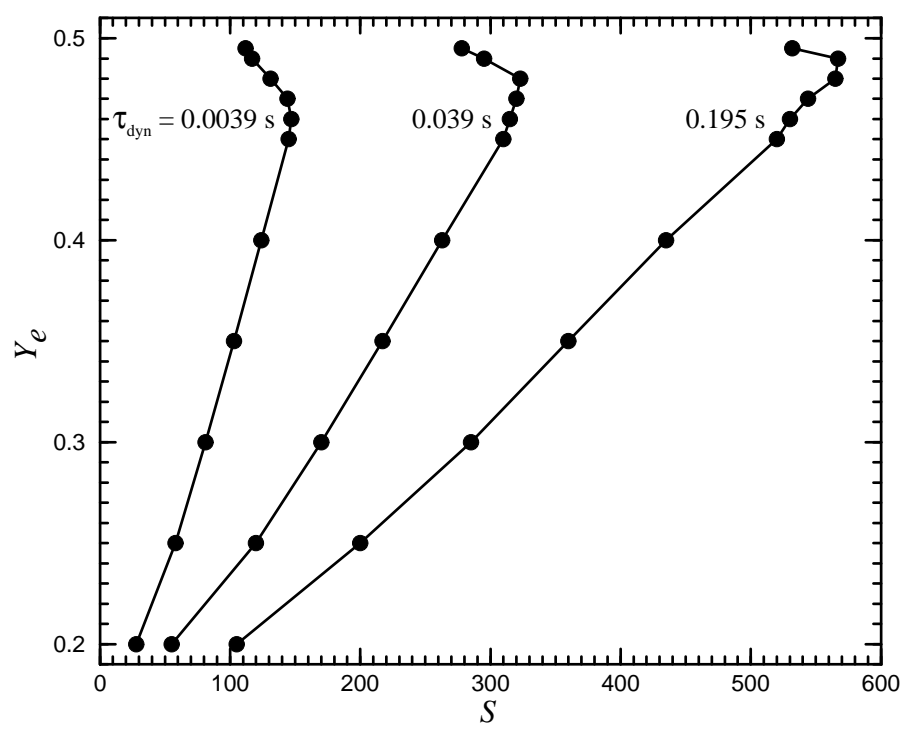

Figure 2: Combinations of $Y_{e}$ and $S$ that would result in the major production of the heavy $r$-elements with $A>130$ during the adiabatic expansion of matter from a hot and dense initial state for three values of $\tau_{\mathrm{dyn}}$. See [10] for details.

dynamic timescale, the evolution of $\rho$ can be determined from the conservation of entropy $S(T, \rho)$ (e.g., $S \propto T^{3} / \rho$ for radiation-dominated matter). In this case, the nucleosynthesis is governed by the three parameters $Y_{e}, \tau_{\mathrm{dyn}}$, and $S$ of the expanding matter. The combinations of $Y_{e}$ and $S$ that would result in the major production of the heavy $r$-elements with $A>130$ for three values of $\tau_{\mathrm{dyn}}$ [10] (see also [11, 12]) are shown in Figure 2.

The sets of $Y_{e}, S$, and $\tau_{\mathrm{dyn}}$ shown in Figure 2 correspond to approximately the same neutron-tosee ratio $Y_{n} / Y_{s}$ that is required to produce the heavy $r$-elements according to Equation (1.2). This ratio is determined as follows. When the expanding neutron-rich matter cools to $T \sim 0.5 \mathrm{MeV}$, essentially all the initial protons are assembled into $\alpha$-particles. An $\alpha$-process [13] starts to burn the $\alpha$-particles and the remaining neutrons into heavier nuclei as $T$ further decreases. This process must go through the bottleneck of three-body reactions, mainly $\alpha+\alpha+n \rightarrow{ }^{9} \mathrm{Be}+\gamma$. Once the bottleneck is passed, the subsequent reactions starting with ${ }^{9} \mathrm{Be}+\alpha \rightarrow{ }^{12} \mathrm{C}+n$ proceed rather efficiently. The nuclear flow generally has reached the nuclei with $A \sim 90$ by the time all CPRs cease at $T \sim 0.25 \mathrm{MeV}$ due to the prohibitive Coulomb barrier. These nuclei then become the seed nuclei to capture the surviving neutrons during the ensuing $r$-process at lower $T$. Clearly, a lower $Y_{e}$ tends to give more neutrons, i.e., a larger $Y_{n}$ at the end of the $\alpha$-process. A shorter $\tau_{\text {dyn }}$ results in fewer seed nuclei, i.e., a smaller $Y_{s}$ due to the shorter duration of the $\alpha$-process. A higher $S$ also leads to a smaller $Y_{s}$. For the radiation-dominated matter with $S \gg 10$ (in units of Boltzmann constant per baryon), there are many photons on the high-energy tail of the BoseEinstein distribution that are able to incur the reaction $\gamma+{ }^{9} \mathrm{Be} \rightarrow \alpha+\alpha+n$, which requires a photon of only $1.573 \mathrm{MeV}$. Consequently, a high $S$ reduces the production of the seed nuclei by suppressing the abundance of ${ }^{9} \mathrm{Be}$ during the $\alpha$-process. In general, there are an infinite number of sets of $Y_{e}, S$, and $\tau_{\mathrm{dyn}}$ that can result in the same $Y_{n} / Y_{s}$. For example, Figure 2 shows that for a fixed 
$Y_{e}$, a lower $S$ can be combined with a shorter $\tau_{\mathrm{dyn}}$ to give the same $r$-process production.

\subsection{What is blown in the wind?}

A specific model of $r$-process nucleosynthesis in adiabatically expanding matter is associated with core-collapse supernovae (CCSNe) from massive stars. A few seconds after the collapse of the core of a massive star, the shock ultimately responsible for disrupting the star has proceeded far into the envelope. The nascent neutron star formed by the core collapse is cooling by emitting neutrinos. The matter immediately above the neutron star has $T>1 \mathrm{MeV}$ and is dissociated into free neutrons and protons. As the neutrinos stream through this matter, a fraction of the $v_{e}$ and $\bar{v}_{e}$ are captured through the reactions $v_{e}+n \rightarrow p+e^{-}$and $\bar{v}_{e}+p \rightarrow n+e^{+}$, respectively. These reactions not only heat the matter and make it expand away from the neutron star but also interconvert neutrons and protons, thereby determining the $Y_{e}$ of the matter [14]. The neutrino heating becomes inefficient at distances of several neutron star radii, above which the matter expands essentially adiabatically in the form of a neutrino-driven wind with an approximately fixed set of $Y_{e}, S$, and $\tau_{\mathrm{dyn}}$. It was proposed [15] that the $r$-process occurs in such winds and this possibility has been extensively studied (see e.g., [16-20]). The physical conditions in the neutrino-driven winds from typical neutron stars have also been carefully studied (see e.g., [21, 22]). Unfortunately, these conditions with typical values of $Y_{e} \sim 0.4, S \sim 100$, and $\tau_{\mathrm{dyn}} \sim 0.1 \mathrm{~s}$ (see also [23]) do not match those required for the major production of the heavy $r$-elements (see Figure 2).

On the other hand, it was shown that the CPR elements from $\mathrm{Sr}$ to $\mathrm{Ag}$ with $A \sim 88-110$ are routinely produced by the $\alpha$-process in the neutrino-driven winds (see e.g., [10]). In fact, the production of these elements was so overwhelming for the conditions obtained in some CCSN models that it was regarded as a severe problem for such models (see e.g., $[16,18]$ ). However, this overproduction problem arises only for the winds with relatively low $Y_{e}$ values that leave the neutron star at the earliest times. As the $Y_{e}$ of the wind is determined by the competition between $v_{e}$ capture on neutrons and $\bar{v}_{e}$ capture on protons, it is extremely sensitive to the difference between the rates of these reactions. For example, the $Y_{e}$ can be changed substantially depending on whether the effects of the neutron-to-proton mass difference, nucleon recoil, and weak magnetism are included in the calculation of these rates $[21,24]$. These rates also depend on the $v_{e}$ and $\bar{v}_{e}$ luminosities and energy spectra, which are taken from CCSN neutrino transport calculations. It is conceivable that the relatively low $Y_{e}$ values of the earliest winds are caused by the uncertainties in such calculations. As discussed in [3,25], observations of elemental abundances in metal-poor stars show that the CPR elements are not tightly coupled with the heavy $r$-elements. The production of the CPR elements in the neutrino-driven winds is compatible with the non-production of the heavy $r$-elements therein. The detailed chemical evolution of the CPR elements in the early Galaxy is discussed in [26, 27] based on their association with neutron star formation.

\subsection{Where, oh where has the $r$-process gone?}

The question that remains to be answered is where the $r$-process occurs. Some workers have explored the effects of e.g., the neutron star magnetic field [28] on the neutrino-driven winds in the hope of obtaining the conditions required for the major production of the heavy $r$-elements. Others have proposed different sites for the $r$-process within the general context of CCSNe, such as winds 

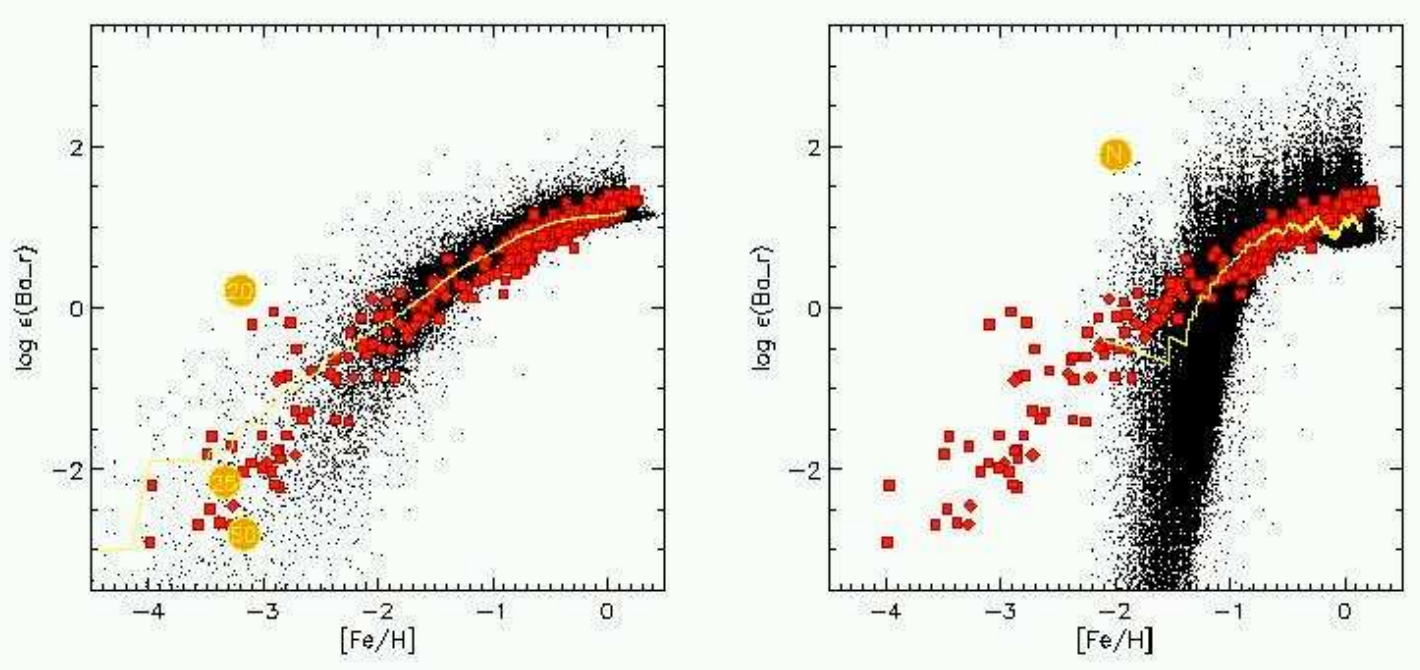

Figure 3: Resulting distribution (dots) of $\log \varepsilon(\mathrm{Ba})_{r}$ vs. [Fe/H] if CCSNe (left panel) or NSMs (right panel) are the major source for the heavy $r$-elements. The data on stars are shown as filled squares. It can be seen that the data strongly disfavor NSMs as the major source for the heavy $r$-elements. See [36] for details.

or jets from accretion disks of black holes (see e.g., [29-31]) or the material undergoing complicated evolution from initial fall-back to final ejection [32]. These studies are focused on obtaining the conditions required for the $r$-process. The approach here is different and uses observations of elemental abundances in stars to constrain the $r$-process site based on considerations of Galactic chemical evolution and a basic understanding of stellar physics. This approach can be illustrated by contrasting CCSNe against neutron star mergers (NSMs; see e.g., [33, 34]) as the major source for the heavy $r$-elements (see e.g., [35, 36]).

The dots in Figure 3 represent the resulting distribution of $\log \varepsilon(\mathrm{Ba})_{r} \mathrm{vs} .[\mathrm{Fe} / \mathrm{H}]$ if CCSNe (left panel) or NSMs (right panel) are the major source for the heavy $r$-elements [36]. Here $\log \varepsilon(\mathrm{Ba})_{r}$ corresponds to the Ba contributed by the $r$-process. As NSMs do not produce Fe, the sources for Fe are the same for both cases. CCSNe are the only Fe source at $[\mathrm{Fe} / \mathrm{H}] \lesssim-1.5$, while both $\mathrm{CCSNe}$ and Type Ia $\mathrm{SNe}$ contribute $\mathrm{Fe}$ at $[\mathrm{Fe} / \mathrm{H}]>-1.5$. Figure 3 shows that there is a sudden rise of $\log \varepsilon(\mathrm{Ba})_{r}$ at $[\mathrm{Fe} / \mathrm{H}] \sim-2$ and wide dispersion in $\log \varepsilon(\mathrm{Ba})_{r}$ at any $[\mathrm{Fe} / \mathrm{H}]$ value for $[\mathrm{Fe} / \mathrm{H}] \gtrsim-2$ if NMSs are the major source for the heavy $r$-elements. This can be understood from the difference in the rate of occurrences between CCSNe and NSMs. The Galactic rates of CCSNe and NSMs are $\sim 10^{-2}$ and $\sim 10^{-5} \mathrm{yr}^{-1}$, respectively. If NSMs were the major source for the heavy $r$-elements, many CCSNe would have enriched an interstellar medium (ISM) with Fe before an NSM provided $\mathrm{Ba}$ to this ISM, which explains the sudden rise of $\log \varepsilon(\mathrm{Ba})_{r}$ at the relatively high value of $[\mathrm{Fe} / \mathrm{H}] \sim$ -2 (right panel of Figure 3). Further, the low Galactic rate of NSMs means that very few NSMs could have occurred in an average ISM over the entire Galactic history [35], which explains the wide dispersion at any $[\mathrm{Fe} / \mathrm{H}]$ value for $[\mathrm{Fe} / \mathrm{H}] \gtrsim-2$ (right panel of Figure 3 ). The filled squares in Figure 3 show the data on $\log \varepsilon(\mathrm{Ba})_{r}$ vs. $[\mathrm{Fe} / \mathrm{H}]$ for stars. It can be seen that the data strongly disfavor NSMs as the major source for the heavy $r$-elements. In contrast, a major CCSN source for these elements is compatible with the data. 

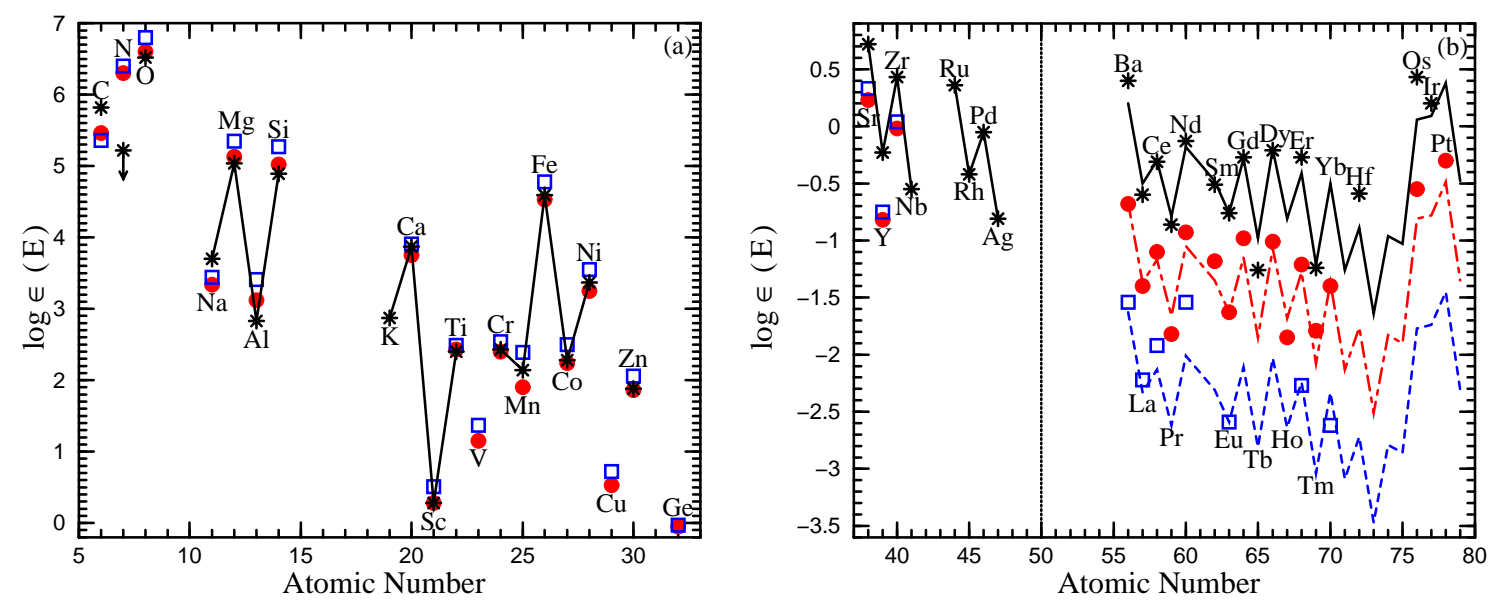

Figure 4: Data on the elements from C to Pt in CS 31082-001 (asterisks; [7]), HD 115444 (filled circles), and HD 122563 (squares; [37]) with $[\mathrm{Fe} / \mathrm{H}]=-2.9,-2.99$, and -2.74 , respectively. (a) The $\log \varepsilon$ values for the elements from C to Ge. The data on CS 31082-001 are connected by solid line segments as a guide. The downward arrow at the asterisk for $\mathrm{N}$ indicates an upper limit. Note that the available abundances for the elements from $\mathrm{O}$ to Ge are almost indistinguishable for the three stars. (b) The $\log \varepsilon$ values for the elements from Sr to Pt. The data for CS 31082-001 in the region to the left of the vertical dotted line are again connected by solid line segments as a guide. In the region to the right of the vertical dotted line, the data on the heavy $r$-elements are compared with the solid, dot-dashed, and dashed curves, which are the solar $r$-pattern [9] translated to pass through the Eu data for CS 31082-001, HD 115444, and HD 122563, respectively. Note the general agreement between the data and the solid and dot-dashed curves. There is a range of $\sim 2$ dex in the abundances of the heavy $r$-elements for the three stars shown.

The above approach also provides important information on the specific characteristics of the major source for the heavy $r$-elements. Figure 4 shows the $\log \varepsilon$ values for the elements from $\mathrm{C}$ to $\mathrm{Pt}$ in three metal-poor stars CS 31082-001 [7], HD 115444, and HD 122563 [37] with [Fe/H] = -2.9, -2.99 , and -2.74 , respectively. The abundances of the heavy $r$-elements in these stars differ by a factor of $\sim 100$ (see Figure $4 \mathrm{~b}$ ). However, they have essentially the same abundances of the low- $A$ elements from $\mathrm{Na}$ to $\mathrm{Ge}$ (including $\mathrm{Fe}$ ) with $A \sim 23-70$ (see Figure $4 \mathrm{a}$ ). This shows that the production of the heavy $r$-elements must be completely decoupled from that of the low- $A$ elements [38]. This decoupling is also demonstrated by the comparison of CS $22892-052([\mathrm{Fe} / \mathrm{H}]=-3.1)$ with HD $221170([\mathrm{Fe} / \mathrm{H}]-2.2)$ and that of CS 31082-001 $([\mathrm{Fe} / \mathrm{H}]=-2.9)$ with $\mathrm{BD}+17^{\circ} 3248$ $([\mathrm{Fe} / \mathrm{H}]=-2.1)$ in Figures 1a and $1 \mathrm{~b}$, respectively. The stars in each pair have essentially the same abundances of the heavy $r$-elements. However, as indicated by the Fe abundances, the abundances of the low- $A$ elements ${ }^{2}$ differ by factors of $\sim 8$ and $\sim 6$ for the paired stars shown in Figures 1a and $1 \mathrm{~b}$, respectively.

The observations discussed above require that the major source for the heavy $r$-elements produce none of the low- $A$ elements. This means that normal CCSNe from massive stars of $\gtrsim 12 M_{\odot}$

\footnotetext{
${ }^{2}$ Observations (see e.g., [39]) show that the abundance patterns of the low- $A$ elements are rather uniform for nearly all the metal-poor stars with $-4 \lesssim[\mathrm{Fe} / \mathrm{H}] \lesssim-1.5$. This is true for the stars shown in Figure 1 in particular. So the Fe abundance reflects the level of enrichment for all the low- $A$ elements in these stars.
} 
cannot be such a source. These stars develop an Fe core surrounded by extensive shells at the end of their lives and produce the low- $A$ elements by the hydrostatic burning in the outer shells during the pre-SN evolution and by the explosive burning associated with the propagation of the SN shock through the inner shells (see e.g., [40]). In contrast, massive stars of $\sim 8-11 M_{\odot}$ develop an $\mathrm{O}-\mathrm{Ne}-\mathrm{Mg}$ core with thin shells of very little mass between the core and the hydrogen envelope (see e.g., [41, 42]). Consequently, the CCSNe from these stars produce a very insignificant amount of the low- $A$ elements, thereby satisfying the observational requirement on the major source for the heavy $r$-elements. This has led to the proposal that these low-mass CCSNe are this major source [43]. A subsequent work further proposed that the heavy $r$-elements are produced as the SN shock rapidly accelerates through the surface layers of an O-Ne-Mg core [44]. However, the conditions adopted by this work are not readily obtained in the current SN models [45] based on the pre-SN structure calculated in $[41,42]$.

\section{Conclusions}

There has been significant progress in the understanding of the $r$-process through both theoretical and observational studies over the last few decades. The conditions required for the major production of the heavy $r$-elements have been derived for a generic astrophysical model where matter adiabatically expands from a hot and dense initial state. Nucleosynthesis in the neutrino-driven winds from nascent neutron stars has been studied extensively. While such winds readily produce the elements from $\mathrm{Sr}$ to $\mathrm{Ag}$ through charged-particle reactions in the $\alpha$-process, they appear incapable of making the heavy $r$-elements. The insights provided by observations of elemental abundances in metal-poor stars are especially important. These observations have demonstrated that the production of the heavy $r$-elements must be associated with massive stars evolving on short timescales, provided evidence strongly favoring CCSNe over NSMs as the major source for these elements, and shown that this source cannot produce any significant amount of the low- $A$ elements from $\mathrm{Na}$ to Ge including Fe. A self-consistent astrophysical model of the $r$-process remains to be developed, and it appears well worthwhile to carry out more comprehensive studies on the evolution and explosion of the massive stars of $\sim 8-11 M_{\odot}$ that undergo O-Ne-Mg core collapse and produce a very insignificant amount of the low- $A$ elements.

\section{References}

[1] J.J. Cowan, F.-K. Thielemann and J.W. Truran, Phys. Rep. 208 (1991) 267.

[2] Y.-Z. Qian, Prog. Part. Nucl. Phys. 50 (2003) 153.

[3] Y.-Z. Qian and G.J. Wasserburg, Phys. Rep. 442 (2007) 237.

[4] M. Arnould, S. Goriely and K. Takahashi, Phys. Rep. 450 (2007) 97.

[5] C. Sneden et al., ApJ 591 (2003) 936.

[6] I.I. Ivans et al., ApJ 645 (2006) 613.

[7] V. Hill et al., $A \& A 387$ (2002) 560.

[8] J.J. Cowan et al., ApJ 572 (2002) 861. 
[9] C. Arlandini et al., ApJ 525 (1999) 886.

[10] R.D. Hoffman, S.E. Woosley and Y.-Z. Qian, ApJ 482 (1997) 951.

[11] B.S. Meyer and J.S. Brown, ApJS 112 (1997) 199.

[12] C. Freiburghaus et al., ApJ 516 (1999) 381.

[13] S.E. Woosley and R.D. Hoffman, ApJ 395 (1992) 202.

[14] Y.-Z. Qian et al., Phys. Rev. Lett. 71 (1993) 1965.

[15] S.E. Woosley and E. Baron, ApJ 391 (1992) 228.

[16] B.S. Meyer et al., ApJ 399 (1992) 656.

[17] K. Takahashi, J. Witti and H.-T. Janka, A\&A 286 (1994) 857.

[18] S.E. Woosley et al., ApJ 433 (1994) 229.

[19] S. Wanajo et al., ApJ 554 (2001) 578.

[20] S. Wanajo and Y. Ishimaru, Nucl. Phys. A 777 (2006) 676.

[21] Y.-Z. Qian and S.E. Woosley, ApJ 471 (1996) 331.

[22] T.A. Thompson, A. Burrows and B.S. Meyer, ApJ 562 (2001) 887.

[23] J. Witti, H.-T. Janka and K. Takahashi, A\&A 286 (1994) 841.

[24] C.J. Horowitz and G. Li, Phys. Rev. Lett. 82 (1999) 5198.

[25] Y.-Z. Qian and G.J. Wasserburg, ApJ 559 (2001) 925.

[26] Y.-Z. Qian and G.J. Wasserburg, ApJ 686, in press (2008) [arXiv: 0807.0809 ].

[27] G.J. Wasserburg and Y.-Z. Qian, in these proceedings.

[28] T.A. Thompson, ApJ 585 (2003) L33.

[29] A.G.W. Cameron, ApJ 562 (2001) 456.

[30] J. Pruet, S.E. Woosley and R.D. Hoffman, ApJ 586 (2003) 1254.

[31] G.C. McLaughlin and R. Surman, Nucl. Phys. A 758 (2005) 189.

[32] C.L. Fryer et al., ApJ 646 (2006) L131.

[33] C. Freiburghaus, S. Rosswog and F.-K. Thielemann, ApJ 525 (1999) L121.

[34] S. Rosswog et al., A\&A 341 (1999) 499.

[35] Y.-Z. Qian, ApJ 534 (2000) L67.

[36] D. Argast et al., A\&A 416 (2004) 997.

[37] J. Westin et al., ApJ 530 (2000) 783.

[38] Y.-Z. Qian and G.J. Wasserburg, ApJ 567 (2002) 515.

[39] R. Cayrel et al., $A \& A 416$ (2004) 1117.

[40] S.E. Woosley and T.A. Weaver, ApJS 101 (1995) 181.

[41] K. Nomoto, ApJ 277 (1984) 791.

[42] K. Nomoto, ApJ 322 (1987) 206.

[43] Y.-Z. Qian and G.J. Wasserburg, ApJ 588 (2003) 1099.

[44] H. Ning, Y.-Z. Qian and B.S. Meyer, ApJ 667 (2007) L159.

[45] H.-T. Janka et al., A\&A 485 (2008) 199. 